\title{
“ENVERDECIENDO” EL CAPITALISMO: UNA GUERRA CONTRA LA SUBSISTENCIA ${ }^{1}$
}

\section{“GREENING” CAPITALISM: A WAR AGAINST SUBSISTENCE}

\author{
Ana Isla*
}

RESUMEN

El capitalismo "verde", como se presenta en las tres Conferencias de las Naciones Unidas sobre el Medio Ambiente y el Desarrollo, se ha propuesto para enfrentar las crisis del medio ambiente (ecológicos) y social (pobreza) que se vive actualmente en todo el mundo. Este "enverdecimiento" es una nueva etapa de acumulación de capital que implica: el uso de mecanismos financieros, como los intercambios de deuda por naturaleza; la licencia de las ONG ambientales para negociar los recursos de los países endeudados con las grandes corporaciones, el establecimiento de los valores monetarios de los "bienes comunes globales". Por otro lado, los nuevos trabajadores son mujeres y hombres campesinos e indígenas que han adquirido nuevos papeles como proveedores de servicios en las nuevas industrias, como el llamado turismo ecológico. En este artículo se considera Costa Rica en esta etapa del capitalismo y se hacen las críticas al respecto.

PALABRAS CLAVE: CAPITALISMO * MEDIO AMBIENTE * CRISIS ECOLÓGICA * FEMINISMO * GRUPOS DE INTERÉS

\section{ABSTRACT}

Green Capitalism, as presented at the three linked United Nations Conferences on Environment and Development, has been proposed as a means to confront the environmental (ecological) and social (poverty) crises currently being experienced around the world. This "greening" is a new stage of capital accumulation that entails: the use of financial mechanisms, such as debt-for-nature exchanges; the license of environmental NGOS to broker the indebted countries' resources with large corporations, and to establish the monetary values of the 'global commons'. Meanwhile, the new labourers are peasants and Indigenous women and men who have acquired new roles as service providers in the new industries, such as eco-tourism. This paper considers this stage of capitalism in Costa Rica and the critiques to it.

KEYWORDS: CAPITALISM * ENVIRONMENT * ECOLOGICAL CRISIS * FEMINISM * INTEREST GROUPS

$1 \quad$ Este artículo se basa en el libro The "Greening" of Costa Rica: women, peasants, indigenous people and the remaking of nature, publicado por University of Toronto Press (Isla 2015). La información fue recolectada por 10 años en diversos lugares en el Área de Conservación Arenal-Tilarán (ahora llamada Área de Conservación ArenalTempisque), donde se realizaron entrevistas y grabaciones en español con 70 miembros de diversas comunidades, varios oficiales del World Wildlife Fund-Canada y del MINAE.

* Brock University, Canadá.

aisla@brocku.ca 


\section{INTRODUCCIÓN}

Las feministas que reflexionan sobre género y medio ambiente, sostienen que ha sido posible mantener la ilusión de que el crecimiento económico es un proceso positivo y benigno, debido a que los costos han sido pagados por aquellas(os) a quienes se ha devaluado: las mujeres, los campesinos, los indígenas, los países periféricos y endeudados, $y$ la naturaleza.

Desde la Cumbre de la Tierra, en 1992, desarrollo y medio ambiente han sido vinculados, nuevas áreas de intervención global se han abierto y la naturaleza ha entrado en el dominio de la política ecológica del neoliberalismo. A este proceso se le llamará greening (enverdeciendo) para indicar cómo, bajo las condiciones del capitalismo verde, los ecosistemas de los países endeudados se han visto crecientemente desestabilizados. Según Mellor (2010), tres condiciones hacen posible el greening:

a) El rechazo de los economistas a reconocer los límites físicos del planeta con el objetivo de continuar con el crecimiento económico.

b) La irracionalidad de los biólogos dedicados al enclaustramiento de la tierra de los países endeudados para negociar con las corporaciones y ponerle precio a los bienes comunes, ahora encerrados en Áreas de Conservación (Ac).

c) El endeudamiento de los países periféricos como resultado de la expansión del capital financiero, y la aplicación del programas de Estabilización del Fondo Monetario Internacional (FMI) y el Ajuste Estructural del Banco Mundial. En este contexto, la venta de la deuda ha sido una enorme fuente de ganancia en la industria del capital financiero.

El propósito de este artículo es desafiar las pretensiones de que el "capitalismo verde" crea igualdad social, reduce la pobreza, confronta la destrucción de la ecología y combate el cambio climático. Este desafío se realiza a través de analizar un estudio de caso sobre el canje de deuda por naturaleza entre Canadá y Costa Rica ( $\mathrm{CDNCCR})$, ejecutada entre 1995 y
1999 por dos Organizaciones No Gubernamentales de Medio Ambiente (оngma) que operaron en el Área de Conservación Arenal (ACA-Tilarán). Se presentan tres crisis que tuvieron efectos muy negativos en la vida de mujeres y niños, campesinos y comunidades indígenas, $y$ en la naturaleza.

\section{EL "GREENING" DEL CAPITALISMO: EL DESARROLLO SUSTENTABLE}

En 1992, en la Cumbre de la Tierra, los gobiernos participantes negociaron un plan de acción, Agenda 21, que oficialmente vinculó el concepto de desarrollo sustentable (Ds), el desarrollo económico y la ecología. Este concepto de ds se equiparó con el crecimiento económico, el cual según los proponentes, podría rescatar a los países pobres desde su pobreza, aun en las áreas más remotas del mundo (Pearce y Warford 1993). Los economistas neoliberales propusieron que la ecología debe estar dentro del sistema económico a través del sistema de precios, en otras palabras, la economía requiere un mundo monetizado para ser protegido. Esto significa que los bienes comunes ecológicos, como la atmósfera, océanos y mares, forestas, montañas, biodiversidad, ecosistemas, agua etc., necesitan tener precio como capital natural (Hamilton 2001); este acuerdo legitimó la privatización de los bienes comunes ecológicos a través del enclaustramiento. La Cumbre de 1992 también le dio al Banco Mundial el poder de avanzar sus prescripciones para manejar la política ecológica del capital global.

La idea del Ds es que tiene que haber un proceso de intercambio entre aquellos que tienen dinero para comprar $y$ aquellos que tienen capital natural para vender. De acuerdo al Banco Mundial, "en el mundo hay un deseo considerable de pagar para preservar la naturaleza y sus funciones críticas que el ecosistema provee" (World Bank 1997, 20). Este punto de vista asume que el bienestar de los humanos es de primera importancia para la economía, a fin de maximizar la satisfacción de los deseos de la humanidad.

El ecofeminismo critica al Ds dentro del marco de crecimiento económico porque los 
programas asociados con Ds resultan en enclaustramiento y housewifization (domesticación). El enclaustramiento es el cercado de los bienes comunes (tierra, agua, foresta, montañas, etc.) y la eliminación de los derechos establecidos por las costumbres de la gente que vive en el lugar. De acuerdo con Hobsbawm, el enclaustramiento se inició en Inglaterra cerca del 1500 (1996, 31).

Silvia Federici (2009) describe que la "Caza de Brujas" en Europa en esos siglos fue un proceso de devaluación de las mujeres como parte del enclaustramiento de los bienes comunes. Con el enclaustramiento de los comunes, los aspectos sociales de la comunidad y su autonomía fueron eliminados. El capitalismo no podría ser tan poderoso sin el control de las mujeres, $y$ esto no podría haber ocurrido sin el "holocausto femenino" que fue la "Caza de Brujas". El capitalismo produjo una nueva División Sexual del Trabajo (DST), en el que la posición de las mujeres como proveedoras en la sociedad fue definido en relación a los hombres, $y$ las convirtieron en esposas, hijas, madres $y$ viudas. Todos esos nombres "ocultan su status de trabajadoras, mientras se da libre acceso a los hombres al cuerpo de las mujeres y su trabajo, y también al cuerpo y trabajo de sus hijos" (Federici 2009, 182-183). Con la llegada del capitalismo, el trabajo reproductivo de las mujeres se puso al servicio de un sistema de acumulación internacional, en el que los vientres de las mujeres se han convertido en propiedad pública $y$ ellas son forzadas a funcionar como un instrumento para la reproducción del trabajo y la expansión de la fuerza de trabajo, como territorio común de los hombres y el Estado (Federici 2009, 170). En esta División Sexual del Trabajo, las mujeres fueron forzadas a un estado de trabajo reproductivo (o doméstico): como recursos, devaluadas, dependientes y perversas.

El concepto de housewifization o domesticación es resultado del prejuicio europeo que asume que el trabajo de las mujeres no tiene valor y que el trabajo no pagado tampoco tiene valor. Cuando mujeres, campesinos e indígenas son descritos como "cerca de la naturaleza", Maria Mies (1986) sostiene que ellos son devaluados. La devaluación se aplica a los sectores económicos marginados o externalizados, como los campesinos e Indígenas cuando sus tierras y productos son apropiados sin o muy poca compensación a través de la violencia estructural.

Vandana Shiva $(1989,10)$ deja al descubierto otro prejuicio cultural del Occidente, quienes crearon el mito de la subsistencia como pobreza. Ella sostiene que las economías de subsistencia que satisfacen las necesidades básicas a través de auto-aprovisionarse no son pobres en el sentido de estar privados. Esas economías han sido universalmente denominadas pobres después de la Segunda Guerra Mundial. Marilyn Waring (1988) sostiene que el Producto Nacional Bruto, que solo cuenta bienes y servicios que pasan a través del mercado, fue introducido como estándar internacional para medir la riqueza de las naciones. Pero valorando la riqueza en dinero, bajo la teoría del costo $y$ beneficio solamente, deja invisible la riqueza de la naturaleza y las habilidades de producción de las mujeres, campesinos e indígenas.

Muchas ecofeministas académicas ven en las economías de subsistencia el nuevo modelo social, económico, de género y de justicia de medio ambiente (Mies y Shiva 1993; Salleh 1997). Por ejemplo, Veronika Bennholdt-Thomsen y Maria Mies $(1999,20)$ proponen la "Perspectiva de la Subsistencia" basada en la producción que "incluye todos los trabajos que participan en la creación, re-creación y mantenimiento inmediato de la vida y otros propósitos". Para ellas, "producción de subsistencia... contrasta con la producción de plusvalor para la ganancia" (1999, 20 ). Las autoras han propuesto una forma de economía que resista los efectos del capitalismo global y reconozca que la vida viene de las mujeres y la comida viene de la tierra (1999). En el mundo entero, la mayoría de productoras de subsistencia son mujeres, por lo que ellas deben ser vistas como expertas $y$ pueden ayudar a recuperar la autonomía de la vida, empezando por la autonomía territorial, seguridad alimentaria en pequeñas granjas y energía eficiente.

\section{EL GREENING EN COSTA RICA: LA POLÍTICA ECOLÓGICA DEL NEOLIBERALISMO}

La crisis de la deuda de 1981 llevó al FMI, al Banco Mundial y a la administración del Presidente Ronald Reagan a las puertas 
de Costa Rica (Sojo 1992). Durante la década de la crisis de la deuda externa de los años 80, el gobierno de Costa Rica aceptó la reconfiguración de su economía basado en grandes requerimientos del capital para ganar divisas con el fin de pagar su deuda externa. Este proceso organizó una nueva política de expropiación de la tierra, aceleró la concentración en grandes terrenos comerciales y fue responsable por los cambios en actividades orientadas al servicio, en vez de la agricultura (Costa Rica 1998).

El endeudamiento también fue usado por los Estados Unidos para imponer la autoridad de la Agencia de Estados Unidos para el Desarrollo Internacional (usaid por sus siglas en inglés) como agente directo y tomar control de la economía costarricense (Petch 1988). Durante su permanencia, la política ecológica neoliberal fue manufacturada por el World Wildlife Fund de Estados Unidos (wwF-us) en la Conferencia sobre Estrategia de Conservación para el Desarrollo Sostenible (ECODEs) en 1988. El Biólogo Christopher Vaughan, jefe del wwF-us, enfatizó el rol del crecimiento de la población y de los campesinos en acelerar la degradación del medio ambiente (Quesada 1990). $\mathrm{Su}$ argumento fue que la biodiversidad local estaría seriamente amenazada si el paisaje social y ecológico no se transformaba en áreas de conservación. Los participantes de Ecodes reconocieron que en Costa Rica, la búsqueda del desarrollo como crecimiento económico y la acumulación de capital y tecnología fueron la causa de la crisis de la deuda, la pobreza, el despojo y la deforestación. Sin embargo, crecimiento económico, acumulación de capitales y tecnología, basados en la "ciencia de la naturaleza" fueron ofrecidos como curación en la forma de Ds (Quesada 1990). En Ecodes se vendió la idea de que el canje de deuda por naturaleza es un instrumento de conservación para desarrollar un modelo de manejo del medio ambiente en áreas de conservación que pueden ser aplicadas en todas las ecologías de los países periféricos endeudados del mundo. El Ds, usando el intercambio de deuda por naturaleza, supuestamente traería riqueza, progreso, logros sociales, protección del medio ambiente, y también se pagaría la deuda externa.
En la Cumbre de la Tierra de 1992, el Ds fue declarado responsabilidad de los gobiernos. Costa Rica recibió un premio como ejemplo del desarrollo sustentable en la periferia. El rol de Costa Rica ha sido el de establecer en el mundo periférico endeudado el concepto de áreas de conservación, es decir, el de conectar su naturaleza y sus trabajadores al poder internacional de los acreedores. En efecto, Costa Rica se convirtió en el primer proyecto verde del neoliberalismo.

Un megaproyecto, el Sistema Nacional de Áreas de Conservación (sinac) fue implementado para manejar el país. El sinac dividió el país en 11 áreas de conservación y creó el Ministerio de Ambiente y Energía (minaE). Una de esas es el Área de Conservación Arenal (ACATilarán) cuyo nombre ha sido cambiado por Arenal-Tempisque Área de Conservación. Este es el estudio de caso que ilumina varios de los aspectos del greening.

\section{CANJE DE DEUDA POR NATURALEZA ENTRE CANADÁ Y COSTA RICA}

Para remodelar la naturaleza en áreas de conservación en la periferia endeudada, varios canjes de deuda por naturaleza privadas fueron firmadas (Sheikh 2007 y 2010). Se ilustra el caso del Canje de Deuda entre Canadá y Costa Rica, que es una iniciativa bilateral que implementa programas de Ds en AcA-Tilarán. Los gobiernos canadiense y costarricense no tienen permitido recibir títulos de la deuda directamente, estos tienen que ser donados a las ONGMA, quienes se convierten en acreedores de los gobiernos. Los títulos de esta deuda fueron donados al wwF-Canadá (wwF-C) (una ong del International Union for Conservation of Nature and Natural Resources) $y$ al Instituto Nacional de Biodiversidad (Instituto Nacional de Biodiversidad- INBio 1995).

En aca-Tilarán, el Ministerio de Recursos Naturales, Energía y Minas (mirenem), la Agencia Canadiense de Desarrollo (CIDA) y el Fondo Mundial para la Naturaleza de Canadá (wwF-c) desarrollaron El Plan General de Uso de la Tierra o Plan de la Tierra (Ministerio de Recursos Naturales, Energía y Minas 1993). El Plan de la Tierra se apropió de los bienes naturales comunes y enclaustró 250000 hectáreas de tierra, de 
las cuales los habitantes locales dependían para su subsistencia. En este canje de deuda por naturaleza hubo muchos enclaustramientos, pero solamente se mencionaran tres: la biodiversidad para la biotecnología, la foresta como crédito de carbono y el paisaje para el ecoturismo. Se presentará cada uno de esos enclaustramientos y se explicarán sus resultados.

\section{1) ENCLAUSTRAMIENTO DE LA BIODIVERSIDAD PARA LA BIO-PROSPECCIÓN}

La Convención de la Biodiversidad Biológica (свв) de la Cumbre de la Tierra en 1992, abrió las puertas para la expropiación del material genético por parte de las ongma y corporaciones. La Convención estableció leyes y regulaciones para beneficiar "a toda la humanidad", un eufemismo para referirse a los países industriales capaces de expropiar los componentes bioquímicos de la biodiversidad usando la biotecnología. La biotecnología es el uso de procesos biológicos en la producción industrial. Como resultado del sistema de patentes o Derechos de Propiedad Intelectual (DPI), los laboratorios de las grandes multinacionales están autorizados con el fin de apropiarse de semillas $y$ crear bancos genéticos para acumular, preservar biodiversidad $y$ disfrutar del monopolio de su explotación comercial.

Diferentes niveles de despojo y expropiación de la tierra son necesarios para establecer un amplio terreno para la investigación científica de las corporaciones. En Costa Rica, desde 1998, la Ley de Biodiversidad (nro. 7788, art. 6) es el instrumento judicial para la aplicación del Convenio de Diversidad Biológica firmado en 1992 y ratificado en 1994. Esta ley determinó que las propiedades genéticas y bioquímicas de la biodiversidad silvestre y doméstica están en el dominio público, por lo tanto, el gobierno autoriza la exploración, investigación, bioprospección, uso y explotación de los elementos de la biodiversidad. Desde entonces, el acceso a las especies silvestres se regula a través de licencias, permisos y subastas. El gobierno ha creado la Comisión Nacional para el Manejo de la Biodiversidad (conageBio) para desarrollar y coordinar las políticas de la biodiversidad.
En el 2004, los Derechos de Propiedad Intelectual fueron expandidos con la implementación de los Acuerdos del Tratado de Libre Comercio entre Estados Unidos y Costa Rica. En este, los permisos para la bio-prospección son definidos como "acuerdos de inversión" para concluir que las inversiones deben ser protegidas por los derechos de propiedad intelectual, esto es la privatización y monopolización de los resultados (Rodriguez 1995). La bio-prospección, ingeniería genética y otros sistemas genéticos que investigan y clasifican la biodiversidad para fines comerciales, buscan nuevas muestras potencialmente beneficiosas para las corporaciones, NGO y organizaciones de ayuda internacionales. Esas organizaciones buscan nuevos usos de la biodiversidad y sus productos, monopolizan la información generada $y$ crean barreras que limitan el uso de la biodiversidad en las comunidades locales.

\section{2) ENCLAUSTRAMIENTO DE LA FORESTA COMO SECUESTRADOR DE CARBONO}

Los gobiernos acordaron abordar el cambio climático en la Cumbre de la Tierra en Rio, 1992. Las teorías científicas han ilustrado el hecho de que la vegetación de la foresta absorbe y guarda carbón, de caso contrario el calor se quedaría atrapado en la atmósfera, incrementando la temperatura y acelerando el cambio climático (Lohmann 2005). En la Conferencia sobre Cambio Climático, en Kyoto en 1997, los países industriales propusieron la creación de mecanismos para reducir las emisiones de gas. La venta de oxígeno desde la foresta, que debe absorber dióxido de carbono de los residuos que se desbordan en los países industriales, se convirtió en parte de la agenda del Ds.

El Protocolo de Kyoto fue el seguimiento de United Nations Framework Convention on Climate Change (unfCcc), en la cual se estableció una "solución" no vinculante para estabilizar las emisiones para el año 2000 al nivel de las del año 1990. Entre las seis clases de gases que deben reducirse se encuentra el dióxido de carbono, descargado desproporcionadamente por el mundo industrial; no obstante, reducir las emisiones de este implica un alto costo para las industrias. Las grandes corporaciones, 
con el apoyo de sus gobiernos, propusieron una solución: crear un mercado global de dióxido de carbono y oxígeno, centrado en la foresta de los países endeudados.

De acuerdo al esquema de la Convención del Cambio Climático, países o industrias que reducen sus niveles de emisiones de carbono a los límites designados, podrían vender sus créditos a otros países o industrias que exceden sus niveles de emisión. Acto seguido al Protocolo de Kyoto, el Fondo de Desarrollo Limpio se convirtió en el Mecanismo de Desarrollo Limpio. Esto ha eliminado la responsabilidad de los países industriales a reducir sus niveles de emisión. Este tipo de solución les permite continuar polucionando, debido a que ellos pueden comprar créditos de carbono desde los países endeudados con densa selva tropical.

Desde Kyoto, las selvas tropicales han obtenido valor económico en relación al monto de carbono que pueden secuestrar. Como la emisión de carbono se compra y vende en el mercado, la selva de Costa Rica tiene valor como sumidero de carbono. Costa Rica fue el primer país en organizar el paquete del Joint Implementation Program organizado por las Naciones Unidas (unfccc 2005). Costa Rica voluntariamente colaboró en la venta de crédito de carbono para que el mundo industrial alcance la reducción de sus emisiones. Desde entonces, numerosos pagos por servicios de las selvas tropicales se han creado, entre ellos están: Reducción de Emisiones por Deforestación y Degradación de los bosques (REDD+, por sus siglas en inglés, Reduction of Emissions from Deforestation and Forest Degradation) y el Régimen de Comercio de Derechos de Emisión de la Unión Europea (Eu ETs, por sus siglas en inglés, The European Emissions Trading Systems). Mientras tanto, las emisiones de energía en los países industriales, producidas por carbón, petróleo y gas, proceden sin trabas.

\section{3) ENCLAUSTRAMIENTO DEL PAISAJE PARA EL ECOTURISMO}

Entre otras áreas de intervención esta la conexión entre el turismo y la naturaleza, o lo que se llama ecoturismo. Las ongma, organizadas por los hombres blancos, apuntaron al enclaustramiento de "áreas silvestres" para la recreación. El ecoturismo es promovido como una actividad que contribuye al crecimiento económico y genera ingreso a las comunidades locales mientras protege el medio ambiente. Es aprobado como una actividad amigable, sustentable y que promueve visitas a áreas naturales no perturbadas para estudiar, admirar $y$ apreciar el paisaje, las plantas $y$ animales de los campos, y otros aspectos culturales como las plantas medicinales (Ulloa 1996). Asimismo, ofrece selvas, parques, sol, playas, montañas y conocimiento indígenas. Los promotores del ecoturismo prometen beneficios estéticos y recreacionales para restaurar la salud física, emocional y espiritual del visitante. Ofrecen un mundo de disfrute, libertad, buen gusto, sin riesgos para aquellos con dinero para gastar, simultáneamente ofrecen avanzar económicamente a los grupos más marginados, a los campesinos, mujeres rurales y comunidades indígenas.

Para los gobiernos nacionales y los acreedores internacionales, el ecoturismo significa en general una reputación positiva en la industria de la conservación de la naturaleza y el ingreso de divisas para pagar la deuda externa. El ecoturismo es clasificado como una industria de exportación, porque típicamente los mercados internacionales están considerados para generar divisas e inversiones.

Bajo la presión del fmi y el Banco Mundial, Costa Rica adoptó el ecoturismo como una estrategia de desarrollo económico y empleo con la esperanza de atraer divisas e inversiones para pagar su deuda. Además, el ecoturismo significa en general una reputación positiva en la industria de la conservación. Para los acreedores internacionales, el ecoturismo es clasificado como industria de exportación, porque se dirige a generar divisas e inversiones. Desde principios de 1990, se ha convertido en el destino más importante en América Latina.

En ACA-Tilarán, el Volcán Arenal se ha convertido en el centro del ecoturismo. Con el tiempo, la construcción de hoteles, cabinas y alojamientos han convertido al volcán, montañas, ríos, selvas y bosques en valores económicos que han sido empaquetados, 
productos de marca $y$ vendidos como productos de recreación. Las comunidades que residen en el área, simultáneamente han sido convertidas en productos para el consumo de los turistas en variedad de formas. Algunos miembros se han convertido en especialistas en observar aves, guías de turismo de aventura, mientras que otras son camareras sirviendo licor, sirvientas tendiendo camas o convertidas en productos de marca para los clientes con dinero.

Las áreas agrícolas se convirtieron en áreas para el ecoturismo. Hasta 1990, la mayoría de las comunidades rurales del área trabajaban en agricultura. La tierra es productiva debido al Volcán Arenal que almacena ceniza rica en minerales $y$ nutrientes. Sin embargo, la desregulación de los instrumentos tradicionales que protegían a la agricultura ha hecho la declinación de la producción agrícola. Costa Rica ahora importa cerca del $80 \%$ de sus principales cultivos - arroz y frijoles- que antes producía por sí mismo. El país está perdiendo las 35 variedades de frijoles. Más aun, como el arroz y los frijoles son importados, los campesinos, ahora trabajadores en servicios, se ven obligados a comprar sus productos alimenticios a precios fijados de acuerdo a los caprichos del mercado internacional (cNp 2009).

\section{LAS TRES CRISIS DEL CAPITALISMO VERDE}

Las prácticas pasadas y actuales del desarrollo económico, así como, los proyectos del desarrollo sustentable han sido instrumentos usados por el capitalismo patriarcal para cometer violencia en contra de las mujeres y la naturaleza. Si la naturaleza tiene que ser violentada para el beneficio capitalista, entonces las mujeres $y$ hombres campesinos e indígenas, a quienes se les define como naturaleza, son igualmente violentados.

\section{1) EL GREENING DE LA BIOTECNOLOGÍA EN LOS PUEBLOS INDÍGENAS Y LOS CAMPESINOS}

La Convención de la Biodiversidad y el Gobierno de Costa Rica devaluaron los conocimientos de los bienes comunes de los pueblos indígenas, en su carrera por incrementar el PNB para pagar su deuda. La bio-prospección de la biotecnología ignora la propiedad colectiva, la cultura y el conocimiento de las comunidades, $y$ desconoce el hecho de que ellos han disfrutado $y$ usado la biodiversidad por miles de años. Por lo que, la bio-prospección se ha convertido en bio-piratería. La bio-piratería consiste en la apropiación de los conocimientos tradicionales de los recursos bio-genéticos de los pueblos indígenas y campesinos con el objetivo de alimentar el sistema de conocimientos de las corporaciones colonialistas (Shiva 1999). Para los pueblos indígenas, bio-piratería es la más peligrosa y ofensiva forma de expropiación porque ataca el centro de su vida y su sobrevivencia.

En 1996, para cumplir con la Carta de Intención para el Desarrollo Sustentable, Cooperación e Implementación Conjunta del Protocolo de Kyoto, el Presidente José María Figueres firmó un decreto conocido como Ley Forestal (nro. 7575) y puso en efecto el art. 2 de expropiación de la tierra. Desde entonces, actos de despojo y robo están dentro del marco de iniciativas para avanzar en la conservación. El proyecto del Estado de vender créditos de carbono significó la expropiación de pequeños y medianos dueños de tierras, en muchos casos sin compensación. En 1999, de acuerdo a $L a$ Nación, el gobierno debía más de usd $\$ 100$ millones a los expropiados. En esos tiempos, el gobierno ofreció pagar usd $\$ 6703,45$ por hectárea (Vizcaíno 1999). En el 2001, la Ministra de Ambiente, Elizabeth Odio, abiertamente admitió no haber pagado por la expropiación de la tierra.

Un símbolo de orgullo de los costarricenses, los parques nacionales constituyen un modelo único en el mundo, que ofrecen innumerables beneficios a la sociedad en particular, $y$ al planeta en general, pero están en una situación crítica debido a la falta de recursos para darles sostenibilidad y cancelar la deuda con los antiguos propietarios cuyas tierras fueron expropiadas o congelados por el bien de la conservación (Odio 2001).

En 1996, la Ley de Expropiación dejó campesinos sin tierra $y$ sin dinero, comunidades que vivían del bosque fueron declarados 
enemigos de la foresta. Los campesinos entendieron que sus tierras fueron expropiadas por el gobierno para recibir pequeños montos de dinero del mercado internacional, mientras ellos han sido abandonados.

El desalojo de los habitantes de la selva (indígenas y campesinos) también fue justificado por las afirmaciones de que las personas desplazadas encontrarían empleo en las ciudades. Pero las mujeres costarricenses rurales saben que la ideología donde las ciudades supuestamente ofrecen ricas oportunidades de empleos bien remunerados y la movilidad ascendente es un mito. Al salir de las zonas rurales, se unen a los desempleados en las ciudades, quienes son ignorados por el gobierno.

\section{2) EL GREENING DE LA FORESTA COMO CRÉDITO DE CARBONO}

Uno de los peores efectos del greening es la crisis de la naturaleza. El Gobierno de Costa Rica, a través del minae, evalúa la capacidad de las explotaciones forestales privadas para vender créditos de carbono, especialmente promovidos por empresarios agrícolas a gran escala en asociación con el capital internacional. Esta madera producida comercialmente ha fomentado el establecimiento de plantaciones de árboles de monocultivo. Terrenos clasificados como reservas forestales, que reciben pagos por servicios ambientales, están exentos de impuestos sobre la propiedad. La desgravación fiscal, bajo un esquema denominado Incentivos Forestales Fiscal, subsidia plantaciones de propiedad del capital internacional para promover las especies forestales extranjeras de alto rendimiento y gran aceptación en el mercado, tales como melina (Gmelina arborea utilizado por Stone Forestal, una empresa estadounidense) y teca (Tectona grand utilizado por Bosques de Puerto Carrillo, otra empresa estadounidense) y Maderas de Costa Rica SA (o MACoRI, ahora Preciosa Madera Ltd., una empresa suiza), siendo estos árboles nativos del sur y el sudeste de Asia. Mono-arboricultura se ha definido en este sistema como "reforestación" a pesar de que estas plantaciones constituyen ecosistemas artificiales y a las empresas se les permite cortar los árboles después de 15 años de crecimiento $y$ transformarlos en madera para suelos, papel, muebles o cajas de frutas de exportación. Con crédito otorgado por el Banco Mundial, el Gobierno de Costa Rica promovió la conversión de los ecosistemas forestales en los monocultivos estériles mediante la plantación de bosques homogéneos (Baltodano 2004; Figuerola 2005).

El monocultivo de especies de árboles se ha convertido en una bomba de tiempo para la biodiversidad en Costa Rica. El bosque natural de los trópicos húmedos es un ecosistema altamente productivo, por ejemplo, una hectárea de bosque tropical tiene más de trescientas especies de árboles. Biodiversidad significa que un bosque tendrá un gran número de leguminosas (árboles, arbustos y plantas) con hojas de diferentes tamaños, que reducen el impacto de la lluvia y evitan la erosión. La ingeniera forestal explica cómo las plantaciones de teca han dado lugar a la erosión de los llanos:

Desde la siembra de estas especies foráneas, he observado que la teca tiene un sistema de raíces que crece profundamente en el suelo, pero en la selva los sistemas de nutrientes y la absorción de agua se encuentran en la superficie. En general, los nutrientes y el agua se concentran a una profundidad de entre 70 y 100 centímetros. Como resultado, árboles de teca están rodeados por el suelo en copos. Además, cuando llueve, el gran tamaño de la hoja acumula grandes cantidades de agua que luego se vierte violentamente sobre el suelo. Una gota de agua, a un nivel microscópico, forma un cráter; cuando el agua cae desde 15 metros o más forma agujeros. La caída de agua en el suelo blando destruye el suelo. La propagación de largo alcance de las raíces y de la sombra producida por las hojas obstruye el crecimiento vegetativo en la capa más baja de bosques, lo que podría prevenir el daño del suelo de las cascadas violentas (Torres, entrevista por Isla, agosto de 2000).

Ecologistas de Costa Rica se oponen al pago de servicios ambientales al monocultivo arbóreo, porque no es un bosque: un monocultivo 
no se reproduce por sí mismo, sino que necesita insumos externos, tales como agroquímicos para su crecimiento (Shiva 1989). Muchos de ellos no estaban en contra de la venta de servicios ambientales; en cambio, ellos promueven la reforestación a través de la regeneración natural y simple de los bosques secundarios, que conservan la biodiversidad y regulan hidrología (Figuerola 2003; Franceschi 2006). Ellos argumentan que la conservación de los bosques con especies de maderas nativas, plantas y la fauna asociadas, debe ser una prioridad, que la restauración y la regeneración natural, con su propia complejidad ecológica, es un objetivo legítimo y que a los campesinos locales se les debe tomar en cuenta para evitar conflictos irreconciliables.

\section{3) EL GREENING DE LAS MUJERES Y LOS NIÑOS PARA EL ECOTURISMO}

Las mujeres y los niños afectados por la crisis presentada en este artículo, son tanto campesinos como provenientes de los pueblos indígenas. Para estas mujeres, la desaparición de los bosques es una cuestión de supervivencia, obligándolas a emigrar a San José, capital de Costa Rica, y a las zonas ecoturísticas con la esperanza de obtener un ingreso para ellas $y$ sus familias expropiadas. Introducidas en la economía monetaria, algunas mujeres pobres no tienen más opción que ganar todo o parte de su vida como prostitutas.

A medida que el ecosistema se desintegra, este tiene poderosos efectos sobre el grado de opresión que sufren las mujeres y los niños. El ecoturismo vincula las áreas de conservación $y$ promete un mundo de ocio $y$ de libertad para los que tienen dinero para pagar. Al mismo tiempo, el turismo sexual ofrece el cuerpo de las mujeres y de los niños como puros, exóticos y eróticos. Esta imagen de Costa Rica involucra dos aspectos de la economía patriarcal capitalista: la dominación de los acreedores (países centrales) sobre los deudores (la periferia); y la psicología del patriarcado en el que los hombres desarrollan su "masculación" (Vaughan 2007). Masculación es la identidad masculina explotativa creada por el mundo alienado del capitalismo patriarcal sobre los cuerpos feminizados
(Vaughan 2007). Como Costa Rica se empobrece cada vez más por la deuda externa y el enclaustramiento de los bienes comunes, la marca de las relaciones de poder internacionales se estampa en los cuerpos de sus hijos y mujeres.

La mayor parte de los proxenetas que se benefician de la organización del turismo sexual son hombres de los países del Norte Global (Estados Unidos, Canadá, España, Alemania, Italia, entre otros). Jacobo Schifter (2007) estima que había entre 10 000-20 000 trabajadores del sexo en el país, y entre 25 000-50 000 turistas sexuales -él llama "fornicarios" a los clientes regulares- que visitan cada año, donde el $80 \%$ de ellos son ciudadanos estadounidenses. Además, informa que los Estados Unidos se ha convertido en proxeneta de Costa Rica porque el crack y sexo con prostitutas ayuda a estos turistas masculinos y jubilados a afirmar su masculinidad y "escapar de la realidad" del declive económico y social en su país (Rogers 2009). En el Tico Times se publicita varios tipos de prostitución, como las agencias de venta por correo de la novia, de citas y de escolta. Esas agencias tienen como objetivo llegar a los turistas y expatriados. En el Internet, hay cientos de sitios web vendiendo cuerpos de las mujeres y niños costarricenses. Lamentablemente, Schifter concluye:

Obviamente, la globalización nos ha vinculado a una economía internacional en el que cada país encuentra su especialización. En los países latinos, se concentra cada vez más en nuestros genitales. Si en la agricultura y la industria nuestras manos y pies nos daban de comer antes, ahora penes $y$ vaginas lo hacen. En el caso de Costa Rica, nos guste o no, el turismo sexual es un componente importante de nuestro Producto Nacional Bruto $(2007,265)$.

Como Costa Rica se desliza en una posición subordinada a nivel internacional, el país entero se convierte en un paraíso para el tráfico sexual, la pedofilia y la pornografía infantil. Al centrarse en su sexualidad, estos hombres han desmembrado niñas y niños. De este modo, contribuyen a la industria del turismo mundial, 
a la riqueza de las empresas y para las arcas del Estado con el fin de pagar su deuda.

\section{CRITICA AL DESARROLLO SUSTENTABLE DE LA ECONOMÍA VERDE}

Cantidades exorbitantes de deuda y los intereses de los préstamos internacionales conducen a la destrucción de la gente y socavan las economías sostenibles y habitables. Algunos podrían argumentar que el capitalismo "verde" aumenta el Рів $y$ genera beneficios en dinero para los que controlan los nuevos mercados.

Desde el punto de vista del ecofeminismo, el greening es una nueva fase de acumulación de capitales que implica:

a) El uso de un nuevo instrumento financiero, como el canje de deuda por naturaleza. En este modelo, el deudor está obligado a dedicar recursos domésticos para financiar los proyectos ecológicos a cambio de reducir una porción limitada de la deuda externa. Además la deuda debe ser considerada más allá de la habilidad del país para pagarla, por lo que esta es vendida a una fracción de su valor en el mercado secundario donde uno se los inversores compra el título de la deuda desde otro inversor, en vez de comprarle al país emisor.

b) El Banco Mundial, a cargo del Environmental Facility Fund (EFF), ha licenciado a grandes Organizaciones No-Gubernamentales de Medio Ambiente (ongMA) para articular los recursos de los países endeudados con las grandes corporaciones asociadas a la re-estructuración económica y la globalización. El rol de las ongma es establecer el valor monetario de los bienes "comunes globales" en los países periféricos endeudados, como son la biodiversidad, la foresta, el paisaje, las montañas, entre otros y exportar esos números a la bolsa de valores.

c) La nueva naturaleza está localizada en áreas de conservación. Un área de conservación es un dominio designado donde las actividades públicas y privadas se interrelacionan con el fin de manejar y conservar la naturaleza para la acumulación de capital. El concepto de áreas de conservación se expande con la Reducción de Emisiones por Deforestación y Degradación de los bosques (REDD+, por sus siglas en inglés, Reduction of Emissions from Deforestation and Forest Degradation) para incluir el territorio de los pueblos indígenas, que es la última frontera del capital.

d) La estructura de clase se ha cambiado. Los nuevos trabajadores son mujeres $y$ hombres campesinos e indígenas que han adquirido nuevos roles como proveedores de servicios en la nueva industria, como el ecoturismo.

Este proceso en el que la naturaleza es selectivamente monetarizada y convertida en bienes y servicios comercializados en el mercado financiero, es llamado "Economía Verde". Las feministas sostienen que estableciendo valores económicos en la naturaleza, requiere la devaluación de otras formas de existencia social. Por ejemplo, esta devaluación elimina las habilidades agrícolas, convierte los bienes comunes (la foresta, las montañas, el paisaje, el agua, entre otros) en recursos para el capital, el conocimiento de plantas medicinales en ignorancia de las comunidades y en Propiedad Intelectual (PI) de las corporaciones, la autonomía comunitaria empieza a depender de las grandes empresas, así como, los hombres y mujeres se convierten en trabajadores mercantilizados cuyas necesidades requieren la mediación de los mercados.

\section{CONCLUSIÓN}

Este artículo revela la insuficiencia de las soluciones basadas en el mercado para las crisis ecológicas y sociales, y muestra que la guerra contra la subsistencia es la real guerra del capital. Se ha mostrado el "capitalismo verde" como una nueva etapa de acumulación de capital. Capitalismo "verde" no crea la igualdad social, no reduce la pobreza, no enfrenta la destrucción ecológica, ni lucha contra el cambio climático. En vez de eso, el greening se basa en la creencia del economista de que no existen límites físicos al crecimiento económico; y en 
la opinión de los biólogos que han convertido el conocimiento de las comunidades y pueblos indígenas en áreas de secreto y paranoia. En esta etapa, los bienes comunes de la naturaleza fueron encerrados en áreas de conservación, biológicamente simplificados y fragmentados. Como resultado, el greening coloca la economía y la ecología de Costa Rica en los mercados internacionales, sobre la base de los derechos de propiedad intelectual dominada por patentes, el aire renombrado como créditos de carbono y el paisaje que se vende como ecoturismo. Pero estas intervenciones violentas, en el endeudado territorio de Costa Rica, se han apoderado de las condiciones de supervivencia de los trabajadores que no reciben pagos o son pobremente pagados impidiendo que ellos vivan en sus propios términos. Es necesaria una ruptura radical con el capitalismo para producir y mantener la vida en este planeta.

\section{BIBLIOGRAFÍA}

LIBROS

Bennholdt-Thomsen, Veronica y Maria Mies. 1999. The subsistence perspective, beyond the globalized economy. New York: Zed Books.

Federici, S. 2004. Caliban and the witch: women, the body and primitive accumulation. New York: Autonomedia.

Hobsbawm, Eric. 1996. The age of revolution, 1789-1848. New York: Vintage Books.

Mellor, Mary. 2010. The future of money: from financial crisis to public resources. New York: Pluto Press.

Mies, Maria y Vandana Shiva. 1993. Ecofeminism. London: Zed Books.

Mies, Maria. 1986. Patriarchy and accumulation on a world scale: women in the international division of labour. London: Zed Books.

Pearce, David y Jeremy Warford. 1993. World without end: economics, environment and sustainable development. New York: Oxford University Press.

Petch, Trevor. 1988. "Costa Rica". En The dance of the millions: Latin America and the debt crisis, editado por Jacqueline Roddick, 191-215. London: Latin America Bureau.

Quesada Mateo, Carlos. 1990. Estrategia de conservación para el desarrollo sostenible de Costa Rica. San José: Ministerio de Recursos Naturales, Energía y Minas, República de Costa Rica.

Salleh, Ariel. 1997. Ecofeminism as politics. Nature, Marx and the postmodern. London $y$ New York: Zed Books.

Schifter, Jacobo. 2007. Viejos verdes en el paraiso: turismo sexual en Costa Rica. San Jose: Editorial Universidad Estatal a Distancia.

Sheikh, Pervaze. 2007. Debt-for-nature initiatives and the Tropical Forest Conservation Act: Status and implementation. Washington, dc: Congressional Research Service, Library of Congress.

Shiva, Vandana. 1989. Staying alive, women, ecology and development. London: Zed Books.

Shiva, Vandana. 1999. Biopiracy: the plunder of nature and knowledge. New York: South End Press.

Sojo, Carlos. 1992. La mano visible del mercado: la asistencia de Estados Unidos al sector privado costarricense en la década de los ochenta. San José: Ediciones cries.

Ulloa Gamboa, Carlos. 1996. Diagnóstico socioambiental de la unidad territorial priorizada. La Fortuna, San Carlos: Proyecto de Conservación y Desarrollo Arenal, Tilarán, Costa Rica.

Vaughan, Genevieve. 2007. Introducción a Women and the Gift Economy: A radically different worldview is possible, Genevieve Vaughan, 1-38. Toronto: Innana Publications and Education.

Waring, Marilyn. 1988. If women counted: a new feminist economics. San Francisco: Harper and Row.

\section{PUBLICACIONES PERIÓDICAS}

Baltodano, Javier. 2004. "Bosques en reservas del Ida: biodiversidad y manejo. AmbienTico, n 133: 14-17. 
Figuerola, Juan. 2003. "Pago de servicios ambientales a la tala rasa". Ambien-Tico, $n^{\circ}$ 123: 10-11.

Figuerola, Juan. 2005. "Nativos y exóticos pero conservando la biodiversidad". AmbienTico, n ${ }^{\circ}$ 141: 16-17.

Franceschi, Hannia. 2006. "Conflictos socio ambientales intercampesinos por los recursos naturales". Revista de Ciencias Sociales, $n^{\circ}$ 111-12: 37-56.

Lohmann, Larry. 2005. "Marketing and making carbon dumps: commodification, calculation and counterfactuals in climate change mitigation". Science as Culture 3, 14: 203-235. http://dx.doi. org/10.1080/09505430500216783

Odio, Elizabeth. 2001. "Modelo único en el mundo". Al Día. 24 de agosto, 3.

Rodriguez, Silvia. 1995. "Los determinismos mercantiles y tecnocráticos en el 'modelo' de funcionamiento del INBio". Ambientico, $\mathrm{n}^{\circ}$ 32: 11-16.

Rogers, Tim. 2009. "Costa Rica's Sex-Tourism Is Growing". Tico times. 16 de octubre. Accesado el 16 de octubre de 2015 http://ticotimes.com/costa-rica/sexprostitution-growing

Vizcaíno, I. 1999. "Deuda millonaria por las expropiaciones". La Nacion, 29 de agosto.

World Bank. 1997. "Measuring the Wealth of the Nations". Expanding the measure of wealth. Indicators for environmentally sustainable development: pp. 19-39. Washington, Dc: World Bank.

\section{TEXTOS ELECTRÓNICOS}

United Nations Framework Convention on Climate Change. 2005. "Joint implementation (sI)". Accesado el 16 de octubre de 2015. http://unfccc.int/ kyoto_protocol/mechanisms/joint_ implementation/items/1674.php

ENTREVISTAS

Torres, Sonia (ingeniera forestal). Entrevista por Ana Isla, agosto del 2000.
OTROS

Consejo Nacional de Produccion (cNP). 2009. Ficha tecnica frijol: periodo agricola 2008-2009. San Jose: cNP.

Hamilton, Kirk. 2001. Genuine savings, population growth and sustaining economic welfare. Paper presented at Conference on Natural Capital, Poverty and Development, Toronto, Canada.

Instituto Nacional de Biodiversidad (INBio). 1995. "Biodiversidad y desarrollo socioeconómico: un programa de utilización sustentable de los recursos de la biodiversidad de Costa Rica y consolidación del Instituto National de Bioversidad (INBio)". Proyecto presentado al Comité de Fideicomiso para Bioversidad Costa Rica-Canadá. San José: INBio parque.

Ministerio de Economía, Industria y Comercio, Área de Integración Económica y Desarrollo Regional. 1998. Cuadro No 1: Costa Rica importaciones de frijol: Enero-Abril, 1998. San José: Ministerio de Economía, Industria y Comercio, Área de Integración Económica y Desarrollo Regional.

Ministerio de Recursos Naturales, Energía y Minas. Área de Conservación Arenal. Costa Rica. 1993. Plan general de uso de la tierra. Vols. I-IV. San José: Agencia Canadiense de Desarrollo Internacional y Fondo Mundial para la Naturaleza de Canadá.

Sheikh, Pervaze. 2010. Debt-for-nature initiatives and the Tropical Forest Conservation Act: Status and implementation (cRs report for Congress RL31286). Accesado el 16 de octubre de 2015. http://www.cnie.org/NLE/ CRSreports/10Apr/RL31286.pdf

Fecha de ingreso: 06/05/2015 Fecha de aprobación: 17/09/2015 